Revista Thema

\begin{tabular}{l|l|l} 
v.19 & n.3 & 2021
\end{tabular} p.635-655

\section{O saber sobre as Plantas Alimentícias Não Convencionais (PANC) na Agricultura Familiar vinculada à Escola Família Agrícola da Região Sul (EFASUL), Canguçu, RS}

\section{The knowledge about Non-Conventional Food Plants in Family Agriculture linked to the Family Agricultural School of the Southern Region (EFASUL), Rio Grande do Sul state, Brazil}

Reges Echer ${ }^{1}$, Carlos Rogério Mauch², Gustavo Heiden ${ }^{3}$, Fernanda Doring Krumreich ${ }^{4}$

\title{
RESUMO
}

A oferta alimentar no planeta está restrita a poucas plantas. Estima-se que 30 mil espécies poderiam ser utilizadas na alimentação. Porém, este conhecimento vem sendo perdido na medida que as comunidades tradicionais desaparecem. O presente trabalho teve como objetivo conhecer e registrar as plantas alimentícias não convencionais (PANC) que são reconhecidas ou utilizadas por agricultores familiares vinculados à EFASUL. Foram realizadas entrevistas para listar as PANC, bem como as formas de usos e coletas botânicas para a identificação. Foram citadas 129 espécies pertencentes a 55 famílias. As mais citadas foram Ananas bracteatus, Butia odorata, Eugenia uniflora, Psidium cattleianum e Syagrus romanzoffiana. A principal forma de consumo é ao natural, seguida por saladas e sucos. Frutos são a parte mais utilizada, seguido de folhas e flores. O registro destes saberes abre portas para novas pesquisas sobre o uso tradicional da biodiversidade local e avaliações de compostos bioativos. A busca para salvaguardar e divulgar as informações promove e valoriza a cultura e biodiversidade local, contribui com a soberania e segurança alimentar dos povos tradicionais e das futuras gerações.

Palavras-chave: Educação do campo; etnoconhecimento; agricultura familiar; agrobiodiversidade.

\section{ABSTRACT}

The food availability on the planet is restricted to a few plant species. It is estimated that 30 thousand species could be used as a source of food. However, this knowledge has been lost as traditional communities are extinct. This paper aimed to know and register non-conventional food plants recognized or used by family farmers linked to EFASUL, a Southern Brazilian school focused on teaching farmers' sons. Interviews were conducted to list species and ways of using them as well as botanical collections were made for later identification. We recorded 129

\footnotetext{
${ }^{1}$ Universidade Federal de Pelotas - UFPel, Pelotas/RS - Brasil. E-mail: regesecher@hotmail.com

${ }^{2}$ E-mail: crmauch@gmail.com

${ }^{3}$ E-mail: gustavo.heiden@embrapa.br

${ }^{4}$ E-mail: nandaalimentos@gmail.com
} 
species from 55 plant families. The most cited were Ananas bracteatus, Butia odorata, Eugenia uniflora, Psidium cattleianum and Syagrus romanzoffiana. The most common way of consumption is fresh, followed by salads and juices. Fruits are the most commonly used part, followed by leaves and flowers. Registering this knowledge may provide new studies on the traditional use of local biodiversity and the evaluation of bioactive compounds. The will to safeguard and disseminate information promotes and values local culture and biodiversity contributes to the sovereignty and food security of traditional peoples and future generations.

Keywords: Rural education; ethno-knowledge; family farming; agrobiodiversity.

\section{INTRODUÇÃO}

A alimentação humana é baseada numa reduzida oferta de alimentos. Mais de $50 \%$ das calorias que são consumidas no mundo provêm somente de quatro espécies de plantas e $90 \%$ dos alimentos consumidos são provenientes de apenas 20 tipos de plantas. Porém, acredita-se que pelo menos 30 mil espécies de plantas poderiam ser utilizadas para alimentação. (KELEN et al., 2015). A simplificação e monotonia alimentar gerada a partir de uma base homogênea de produção de alimentos que foi adotada nas últimas décadas, aumenta o risco de deficiência de micronutrientes, tornando-se necessária uma modificação nos padrões agrícolas e alimentares, vinculando-os a natureza e a nutrição. A confiança excessiva em algumas culturas básicas, combinadas com baixa diversidade alimentar, é uma das principais causas de desnutrição. (FAO, 2018). Mesmo que essas culturas forneçam calorias suficientes para evitar a fome, elas não fornecem todos os nutrientes necessários para uma dieta saudável. Altos níveis atuais de desnutrição são frequentes devido a dietas sem diversidade. A diversidade alimentar é considerada baixa quando um alto consumo de cereais é acompanhado por uma baixa ingestão de legumes, frutas e hortaliças, os quais poderiam fornecer os micronutrientes e fibras necessários para uma dieta saudável. (LEVY et al., 2010; FAO, 2018).

Enquanto o sucesso de produção na agricultura nas últimas décadas é visto como marco importante, os benefícios desiguais e impactos negativos dessas políticas sobre os recursos naturais tornam-se mais evidentes. A aceleração de degradação ambiental e a mudança climática também afetou negativamente a produção agrícola e a segurança alimentar tornando-a homogênea e insustentável. (FAO, 2010; FAO, 2018). Estimativas globais indicam que mesmo no século $X X I$, um terço da população enfrenta insegurança alimentar e mais de 800 milhões de habitantes do planeta passam fome. Em resposta, agências internacionais, incluindo a FAO (Organização das Nações Unidas para e Agricultura e Alimentação) e a OMS (Organização Mundial da Saúde) avançaram em pesquisas de novos recursos alimentares, dentre eles, plantas silvestres subutilizadas são consideradas uma alternativa potencial para alcançar a segurança nutricional. Além disso, a incorporação de compostos alimentares biologicamente ativos de plantas comestíveis silvestres trará benefícios diretos à saúde. (CHIVANDI et al., 2015; JOSHI et al., 2018).

A biodiversidade de plantas que ocorre no Brasil é a mais rica do mundo, com $15 \%$ a $20 \%$ das espécies do planeta, sendo considerado o país de maior megadiversidade, possuindo a flora mais rica e o maior remanescente de ecossistemas tropicais, perfazendo 46.852 espécies vegetais. (CORADIN et al., 2011; FLORA DO BRASIL, 
2020). Mesmo assim, o atual modelo alimentar é sustentado por uma matriz agrícola baseada na monocultura e agricultura convencional, consequentemente, apresenta um padrão alimentar refém deste sistema e é predominantemente industrializado, contribuindo para o desconhecimento e a baixa utilização de centenas de espécies nativas com potencial econômico e nutricional. (PASCHOAL et al., 2016).

As Plantas Alimentícias Não Convencionais (PANC) são plantas que são na totalidade ou que possuem uma ou mais partes comestíveis, sendo elas espontâneas ou cultivadas, nativas ou exóticas e que não fazem parte diariamente de nosso cardápio. (KELLEN et al., 2015). Madeira et al. (2013) acrescentam que no cultivo destas plantas não existe uma cadeia produtiva estabelecida, elas apresentam distribuição limitada, são em geral mantidas pelos agricultores, sendo muitas vezes espontâneas e fazem parte da alimentação e cultura do local, utilizando a denominação de hortaliças tradicionais. Na literatura mundial podemos encontrar inúmeras definições que se enquadram no contexto de PANC, entre elas os termos em português como "plantas de crise", "plantas emergenciais" ou "plantas de sobrevivência", "plantas alimentícias alternativas", ou ainda no que se refere a um grupo específico, "hortaliças não convencionais" ou "hortaliças tradicionais" na literatura estrangeira podem ser denominadas em espanhol como "yuyos", "plantas silvestres comestibles", "plantas nativas comestibles" ou em inglês "edible wild plants", "wild food" "emergency foods". (RAPOPORT et al., 1999; MADEIRA et al., 2013; KINUPP; LORENZI, 2014). Para Brack (2016), as PANC também podem ser denominadas de plantas alimentícias da agrobiodiversidade, pois estão associadas a distintas culturas resgatando a riqueza étnica e destacando as especificidades das biorregiões e das formas de produção.

Considerando que a flora brasileira é a mais diversa do planeta, é perceptível o enorme potencial de uso em variados sistemas de produção dentro de um paradigma não produtivista, necessário e urgente, sendo o contraponto ao sistema agrícola convencional. O cultivo e a utilização de PANC são crescentes, devendo ser mantidos e associados aos sistemas agroecológicos, em especial aos Sistemas Agroflorestais (SAF's), sob o resguardo dos agricultores familiares e das populações tradicionais. (BRACK, 2016).

Nesta linha, modelos agrícolas tradicionais compostos pela agricultura familiar ainda mantém vivos conhecimentos relacionados a usos da biodiversidade local. A Agricultura Familiar (AF) é definida como aquela que está baseada predominantemente no trabalho da família dentro da propriedade rural e que é complementado pela existência de uma forte correlação entre gestão, trabalho e posse total ou parcial dos meios de produção, presença de sistemas de produção diversificados, de renda agrícola monetária, de auto consumo e pluriatividade. (BOTELHO FILHO, 2005). Unidades de produção familiares (UPF) correspondem a $84 \%$ do total de estabelecimentos agropecuários brasileiros e ocupam $25 \%$ da área agrícola total, absorvendo $75 \%$ da mão de obra ocupada na agropecuária, produzindo $70 \%$ da alimentação consumida no país e respondendo por $38 \%$ da receita agropecuária nacional (IBGE, 2006).

Baseando-se na perspectiva de aumentar a diversidade alimentar, alicerçando-se no conhecimento tradicional que se estabelece a partir da relação com a biodiversidade local, busca-se então explorar o potencial alimentício e bioativo das PANC, que são 
consideradas uma alternativa saudável e sustentável para serem utilizadas como fonte de alimentação, pois muitas delas ocorrem espontaneamente nos mais diversos ambientes, seja em terrenos baldios, plantações, hortas e jardins. (KINUPP; LORENZI, 2014).

Para Rapoport et al. (1999) em inúmeras comunidades agrícolas ou suburbanas o uso de plantas silvestres está sofrendo um processo de abandono. Numerosos fatores sociológicos e ecológicos proporcionaram o abandono destes recursos naturais devido ao fato de que os costumes alimentares nas culturas tradicionais e primitivas dependem da transmissão oral como única via de transmissão, porém esta via é um processo sensível a aculturação e desenraizamento devido a influências da modernização.

Buscando ressaltar a representatividade do saber tradicional mantido pela agricultura familiar regional buscou-se desenvolver a presente pesquisa com a Escola Família Agrícola da Região Sul (EFASUL). A Escola está localizada no município de Canguçu, Rio Grande do Sul e já formou 2 turmas de Técnicos em Agroecologia, filhos e filhas de agricultores familiares, assentados de reforma agrária e quilombolas, muitos em situação de vulnerabilidade sócio-ambiental. Em seu plano de ensino, a EFASUL busca contribuir para a formação de jovens e seus familiares a partir de uma prática comunitária pautada nos princípios da Educação do Campo, Agroecologia e Pedagogia da Alternância, trabalhando com questões relativas ao conhecimento tradicional, à Agroindústria Familiar Rural e ao trabalho cooperativo como forma de ampliar as possibilidades de renda nas Unidades de Produção Familiares e consolidar espaços de trabalho e de organização de coletivos.

Inúmeros fatores ambientais, biológicos e sociais podem influenciar os hábitos e conhecimento de uso das plantas nativas nas comunidades, buscar conhecer os motivos que influenciaram o uso destas espécies pode permitir manter estas informações vivas e evitar a perda de conhecimentos valiosos. (MONTENEGRO; ZAMBRANO, 2019).

Essa relação construída entre comunidades e o uso das plantas do entorno, corresponde ao objeto de estudo da Etnobotânica, a qual busca conhecer e registrar os saberes para que se possa compreender as relações dos diferentes povos e o ambiente, baseando-se nas crenças e culturas ao longo do tempo. Os estudos etnobotânicos buscam entender como as pessoas interagem com o meio ambiente e como utilizam os recursos das plantas para atender as necessidades culturais e físicas. (ALBUQUERQUE et al., 2019).

Através destes estudos torna-se possível registrar e divulgar as informações, pois ainda existe muito conhecimento deste grupo de plantas entre populações que preservam as antigas tradições de colheita e uso, por isso é importante focar atenção às plantas silvestres como recurso natural, alimentar e de potencial interesse econômico. (RAPOPORT et al., 1999). Desta forma pode-se entender melhor como a ação da espécie humana modificou e ainda modifica ecossistemas e outras espécies e como as decisões humanas passadas podem ter afetado a maneira como interagimos com as plantas no presente. (ALBUQUERQUE et al., 2019). 


\section{Revista Thema}

Assim torna-se importante conhecer os saberes etnobotânicos das comunidades tradicionais e ao mesmo tempo formalizar o registro do conhecimento por elas preservado a fim de evitar que informações tão preciosas sejam perdidas.

\section{METODOLOGIA}

O grupo de estudo compreendeu familiares de educandos da EFASUL, agricultoras e agricultores residentes nos municípios de Canguçu, Cerrito e Pelotas, Rio Grande do Sul (Figura 1). Necessitavam ter como critérios, residir na zona rural e ter vínculo com a agricultura, ser familiar de educando da EFASUL, ter idade mínima de 18 anos completos e autorizarem a participação e gravação após a assinatura de duas vias do Termo de Consentimento e Livre Esclarecido, adaptado de Lovatto (2012).

Figura 1 - Área de abrangência do estudo etnobotânico sobre plantas alimentícias não convencionais (PANC) utilizadas ou reconhecidas pelas 21 famílias de agricultoras e agricultores entrevistados bem como a localização das 21 unidades de produção familiares e da Escola Família Agrícola da Região Sul.

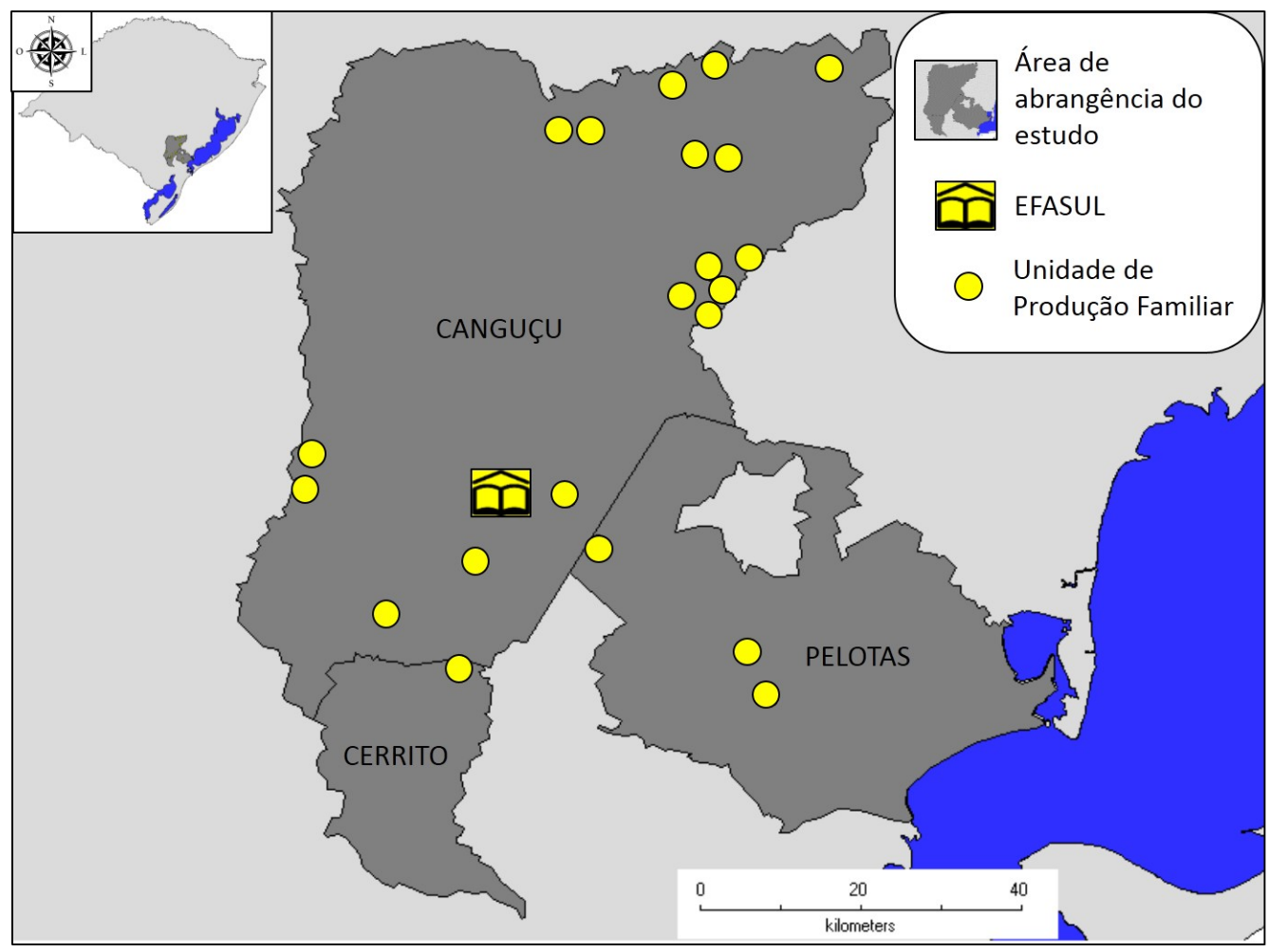

Fonte: Elaborada pelos autores.

O levantamento etnobotânico foi realizado através de pesquisa social-qualitativa por meio de entrevistas semi-estruturadas. (MANZINI, 2003). As entrevistas foram gravadas em gravador de voz digital Novacom R-70 em formato digital mp3 e ocorreram no período de 21 de janeiro de 2019 a 25 de março de 2019.

As perguntas buscaram caracterizar a família e a Unidade de Produção Familiar (UPF), e hábitos relacionados aos usos das plantas. Já referente às PANC, buscou-se saber quais espécies são reconhecidas e utilizadas pelas famílias entrevistadas, quais os 
ambientes de ocorrência, as formas que a planta ou as partes alimentícias são utilizadas e a frequência de uso.

Os critérios adotados para enquadramento como PANC foram baseados em Madeira et al. (2013), Kinupp e Lorenzi (2014) e Brack (2016), ou seja: Plantas que não tenham cadeia produtiva regional estabelecida, apresentam distribuição limitada, são em geral mantidas pelos agricultores, sendo muitas vezes espontâneas e fazendo parte da alimentação e cultura in loco, que não sejam recorrente no uso alimentar, que demandam grandes explicações e que não são convencionais nos cardápios ou não são produzidas pelo sistema agrícola convencional. $O$ uso de partes não convencionais, de plantas convencionais, como por exemplo, folhas da batata-doce ou erva-mate para preparados não convencionais também foram contabilizados. Espécies que agricultoras e agricultores adicionam ao chimarrão foram contabilizadas, uma vez que o chimarrão é entendido como uma bebida ritual, um hábito regional e a infusão de partes não tem a finalidade exclusivamente medicinal, sendo o consumo como estimulante semelhante ao consumo do café. Quando uma espécie de planta foi exclusivamente citada para o preparo de infusões com finalidade medicinal, a planta e as informações relacionadas a ela não foram contabilizadas como alimentícia.

Foram definidas categorias para apontar o reconhecimento e o uso das PANC pelas famílias entrevistadas. Na categoria reconhecimento estão: reconhece o uso, quando o(a) entrevistado(a) sabe que pode ser consumido; plantio recente ainda não frutificou, quando o(a) entrevistado(a) informa que possui a espécie na UPF mas ainda não frutificou e experimentou, quando o(a) entrevistado(a) informa que já experimentou em alguma ocasião. Na categoria de uso estão: consumia, quando o(a) entrevistado(a) consumiu por algum período; raramente consome, quando o(a) entrevistado(a) utiliza de uma a duas vezes por ano; eventualmente consome, quando o(a) entrevistado(a) utiliza de 3 a 5 vezes por ano e por fim, frequentemente consome, quando o(a) entrevistado(a) consome as PANC durante o período de oferta natural e além, através de processados como doce em pasta (Schmier), conservas, doces, compotas, polpa congelada, partes desidratadas entre outros.

Quando disponíveis na UPF, as plantas foram fotografadas e coletadas para herborização para identificação botânica, seguindo os procedimentos do Manual Técnico de Vegetação Brasileira (IBGE, 2012) e o Manual de Procedimentos para Herbário (Peixoto e Maia, 2013).

Para as famílias de plantas adotou-se o sistema de classificação taxonômica Angiosperm Philogeny Group IV (APG IV 2016) e para gêneros e espécies foi seguida a classificação adotada na Flora do Brasil 2020 em construção (2020) ou The Plant List: a working list of all known plant species (The Plant List, 2020) e TROPICOS, do Missouri Botanical Garden. (TROPICOS, 2018). A identificação taxonômica foi realizada por meio de chaves de identificação, e comparação com descrições morfológicas e ilustrações com base na literatura, além de incluir trabalhos que tratam de plantas alimentícias não convencionais, plantas medicinais, frutíferas, daninhas, onde muitas espécies estão contempladas. (KISSMANN 1997 e 2000; KISSMANN e GROTH, 1999; LORENZI e ABREU MATOS, 2008; MOREIRA; BRAGANÇA, 2010; KINUPP e LORENZI, 2014; LORENZI, 2014 e LORENZI et al., 2015). A nomenclatura científica de cada espécie, juntamente com os autores foram padronizados conforme o International Plant Names Index (IPNI, 
2020). Vouchers do material botânico coletado e identificado foram depositados no Herbário ECT da Embrapa Clima Temperado, Pelotas, Rio Grande do Sul.

\section{RESULTADOS}

Foram entrevistadas 21 famílias, sendo a idade média do patriarca de 57 anos, com variação de 38 a 81, enquanto da matriarca de 55 anos, variando de 33 a 82 . 0 tamanho médio das Unidades de Produção Familiares (UPF) foi de 20,38 hectares, variando de 0,46 até 52 hectares, conforme informações relatadas pelos proprietários e o tempo médio de residência nas UPF foi de 27,5 anos, variando de 5 a 73 anos. A escolaridade dos entrevistados foi de 2,5\% com ensino superior e 2,5\% com ensino fundamental completos, $5 \%$ com ensino médio incompleto, $18 \% \mathrm{com}$ ensino médio completo e $72 \%$ com ensino fundamental incompleto. A maioria das famílias é natural da região (70\%) e as demais possuem origem diversificada, do norte e noroeste do Rio Grande do Sul, oeste de Santa Catarina e sul do Paraná. Os entrevistados autodeclararam suas origens étnicas como pomerana, indígena, italiana, espanhola, portuguesa, brasileira, negra, polonesa, francesa, bugra, alemã e austríaca.

A distribuição de uso do solo da UPF, conforme relato das famílias, apresenta média de $38 \%$ destinadas a cultivos anuais, como hortaliças, milho, feijão, mandioca, batata, batata-doce, abóbora, tabaco, melancia, amendoim, morango, moganga e melanciade-porco. Também foi relatado pelos proprietários que $22 \%$ da área total apresenta cobertura de mata nativa, $20 \%$ campos de pastagens e os demais $20 \%$ destinados a cultivos perenes, como frutíferas diversas, dentre as quais: laranja, bergamota, limão, uva, banana, pêssego e goiaba, muitas vezes integrados a Sistemas Agroflorestais, (SAF's) e parte também destinado a infraestrutura de moradia, galpões, estábulos, galinheiros, casas-de-vegetação, estufas de secagem de tabaco, espaço de lazer e estradas internas.

O total de entrevistados afirmam que fazem uso de caules, raízes, tubérculos, folhas, flores e frutos na alimentação de forma alternada, sazonal, porém com frequência e mantém em suas UPF o hábito de ter um espaço destinado especificamente para o cultivo de "hortas caseiras" que além das hortaliças convencionais, apresenta diversidade de conformações com PANC, medicinais, ornamentais, anuais e algumas frutíferas perenes de baixo sombreamento. A totalidade das famílias apresenta produção de hortaliças para autoconsumo.

Quando questionados se já ouviram falar sobre o acrônimo PANC, 13 famílias já tinham ouvido e 8 não. Das que conheciam, tinham ouvido em reuniões do grupo de agroecologia, Pastoral da Saúde, pelos filhos que relataram, por atividades relacionadas à escola EFASUL, por técnicos da EMATER e ainda por reportagem nos meios de comunicação.

As entrevistas apontaram 769 indicações de uso de plantas que se enquadraram no conceito de PANC por todas as famílias entrevistadas quanto ao reconhecimento do uso e do consumo destas e totalizaram 129 espécies de PANC identificadas (Tabela 1), distribuídas em 55 famílias botânicas. 


\section{Revista Thema}

v.19

\begin{tabular}{l|l} 
n.3 & 2021
\end{tabular}

Tabela 1 - Plantas alimentícias não convencionais (PANC) citadas pelas famílias de agricultores familiares entrevistadas, vinculadas à Escola Família Agrícola da Região Sul (EFASUL), Canguçu, Rio Grande do Sul, Brasil.

\begin{tabular}{|c|c|c|c|c|}
\hline Táxons & Nome popular & $\begin{array}{l}\text { Parte } \\
\text { utilizada }\end{array}$ & Forma de uso ou preparo & Voucher \\
\hline \multicolumn{5}{|l|}{ AMARANTHACEAE } \\
\hline Amaranthus deflexus L. & caruru, cururu & folha & saladas/sopas & ЕСТ7430 \\
\hline Beta vulgaris $\mathrm{L}$. & beterraba & folha & sopa/refogada/bolinhos & ЕСT7426 \\
\hline $\begin{array}{l}\text { Pfaffia glomerata (Spreng.) } \\
\text { Pedersen } \\
\text { AMARYLLIDACEAE }\end{array}$ & ginseng-brasileiro & folha & adicionada ao chimarrão & \\
\hline $\begin{array}{l}\text { Nothoscordum gracile } \\
\text { (Aiton) Stearn } \\
\text { ANACARDIACEAE }\end{array}$ & cebolinha-do-campo & $\begin{array}{l}\text { bulbo/caule/ } \\
\text { folha }\end{array}$ & in natura/condimento & ЕСТ7480 \\
\hline Schinus molle L. & aroeira-periquita, mole & $\begin{array}{l}\text { fruto/ } \\
\text { semente }\end{array}$ & condimento & \\
\hline Schinus terebinthifolia Raddi & $\begin{array}{l}\text { aroeira-vermelha, } \\
\text { periquiteira }\end{array}$ & $\begin{array}{l}\text { fruto/ } \\
\text { semente }\end{array}$ & condimento & \\
\hline \multicolumn{5}{|l|}{ ANNONACEAE } \\
\hline $\begin{array}{l}\text { Annona rugulosa (Schltdl.) } \\
\text { H.Rainer }\end{array}$ & $\begin{array}{l}\text { araticum-verde, araticum- } \\
\text { liso }\end{array}$ & fruto & in natura & \\
\hline \multicolumn{5}{|l|}{ APIACEAE } \\
\hline Daucus carota L. & cenoura & folha & $\begin{array}{l}\text { sopa/bolinhos } \\
\text { adicionada ao }\end{array}$ & \\
\hline Foeniculum vulgare Mill. & funcho & folha & $\begin{array}{l}\text { chimarrão/bolos/biscoitos/suc } \\
\text { o }\end{array}$ & ЕСТ7447 \\
\hline Pimpinella anisum $\mathrm{L}$. & erva-doce & $\begin{array}{l}\text { folha/ } \\
\text { semente }\end{array}$ & $\begin{array}{l}\text { adicionada ao } \\
\text { chimarrão/bolos/biscoitos }\end{array}$ & \\
\hline \multicolumn{5}{|l|}{ AQUIFOLIACEAE } \\
\hline Ilex paraguariensis A.St.-Hil. & erva-mate & $\begin{array}{l}\text { folhas } \\
\text { trituradas }\end{array}$ & bolos & ЕСТ7472 \\
\hline \multicolumn{5}{|l|}{ ARACEAE } \\
\hline $\begin{array}{l}\text { antiquorum (Schott) } \\
\text { F.T.Hubb. \& Rehder }\end{array}$ & inhame & rizoma & $\begin{array}{l}\text { cozido/assado/torrado para } \\
\text { fazer bebida tipo café }\end{array}$ & ЕСТ7453 \\
\hline Monstera deliciosa Liebm. & $\begin{array}{l}\text { costela-de-adão, banana- } \\
\text { có }\end{array}$ & fruto & in natura & ЕСТ7424 \\
\hline \multicolumn{5}{|l|}{ ARAUCARIACEAE } \\
\hline $\begin{array}{l}\text { Araucaria angustifolia } \\
\text { (Bertol.) Kuntze }\end{array}$ & pinhão & fruto & $\begin{array}{l}\text { assado/cozido/molho de carne } \\
\text { e triturado para compor } \\
\text { farinha de massa }\end{array}$ & ЕСТ7444 \\
\hline \multicolumn{5}{|c|}{ 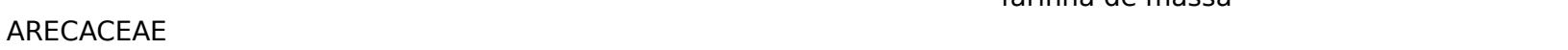 } \\
\hline $\begin{array}{l}\text { Butia odorata (Barb.Rodr.) } \\
\text { Noblick }\end{array}$ & butiá & $\begin{array}{l}\text { fruto/ } \\
\text { amêndoa }\end{array}$ & $\begin{array}{l}\text { in } \\
\text { natura/suco/geleia/schmier/lic } \\
\text { or/amêndoa }\end{array}$ & $\begin{array}{l}\text { ECT7415, } \\
\text { ECT7416, } \\
\text { ЕСT7440, } \\
\text { ECT7441 }\end{array}$ \\
\hline $\begin{array}{l}\text { Syagrus romanzoffiana } \\
\text { (Cham.) Glassm. }\end{array}$ & coquinho, gerivá & $\begin{array}{l}\text { fruto/ } \\
\text { palmito/ } \\
\text { amêndoa/raiz }\end{array}$ & $\begin{array}{l}\text { in natura/amêndoa crua ou } \\
\text { torrada/palmito cru e cozido }\end{array}$ & \\
\hline $\begin{array}{l}\text { ARISTOLOCHIACEAE } \\
\text { Aristolochia triangularis } \\
\text { Cham. } \\
\text { ASPARAGACEAE }\end{array}$ & $\begin{array}{l}\text { cipó-mil-homens, cipó- } \\
\text { milôme }\end{array}$ & caule & infusão gelada & ЕСТ7451 \\
\hline $\begin{array}{l}\text { Asparagus officinalis L. } \\
\text { ASTERACEAE }\end{array}$ & aspargo & rebentos & in natura/salada/cozido & \\
\hline Achillea millefolium $\mathrm{L}$. & mil-em-ramas & folha & adicionada ao chimarrão & ЕСТ7609, \\
\hline $\begin{array}{l}\text { Achyrocline satureioides } \\
\text { (Lam.) DC. }\end{array}$ & marcela, macela & flor & adicionada ao chimarrão & $\begin{array}{l}\text { ЕСТ7419, } \\
\text { ЕСТ7434, }\end{array}$ \\
\hline Baccharis crispa Spreng. & carqueja & folha & $\begin{array}{l}\text { adicionada ao chimarrão/e pra } \\
\text { fazer cerveja }\end{array}$ & $\begin{array}{l}\text { ECT7421 } \\
\text { ЕСT7410, } \\
\text { ЕСT7422, } \\
\text { ECT7428 }\end{array}$ \\
\hline
\end{tabular}


Bidens pilosa L.

Cynara scolymus L.

Galinsoga parviflora Cav.

Helianthus tuberosus L.

Hypochaeris chillensis

(Kunth) Hieron.

Matricaria chamomilla L.

Mikania glomerata Spreng.

Smallanthus sonchifolius

(Poepp.) H.Rob.

Sonchus oleraceus (L.) L

Taraxacum campylodes

G.E.Haglund

BASELLACEAE

Anredera cordifolia (Ten.)

Steenis

BRASSICACEAE

Lepidium didymum $\mathrm{L}$.

Raphanus sativus L.

\section{BROMELIACEAE}

Ananas bracteatus (Lindl.) Schult. \& Schult.f.

Bromelia antiacantha Bertol.

\section{CACTACEAE}

Cereus hildmannianus

K.Schum.

Hylocereus undatus (Haw.)

Britton \& Rose

Opuntia ficus-indica (L.) Mill. figo-da-índia

Opuntia monacantha (Willd.)

Haw.

tuna-palma, palma

Pereskia aculeata Mill.

CANNABACEAE

Celtis iguanaea (Jacq.) Sarg.

CARICACEAE

Vasconcellea quercifolia A.

St.-Hil.

CELASTRACEAE

Monteverdia ilicifolia (Mart.

ex Reissek) Biral

CYPERACEAE

Cyperus esculentus L.

CONVOLVULACEAE

Ipomoea batatas (L.) Lam.

CUCURBITACEAE

Citrullus lanatus (Thumb.)

Matsum. \& Nakai var.

citroides (L.H.Bailey) Mansf.

Cucurbita moschata

Duchesne

Melothria cucumis Vell.

Momordica charantia L.

DIOSCOREACEAE

Dioscorea bulbifera L.

EBENACEAE

Diospyros inconstans Jacq.

EQUISETACEAE

Equisetum arvense $\mathrm{L}$.

picão-branco picão-preto

alcachofra

girassol batateiro

raditi, raditi-de-cachorro

camomila, maçanilha

guáco

yacon, batata-yacon

serralha chicória nativa

dente-de-leão

bertalha

mestruz, mastruço

nabo-forrageiro

ananás, abacaxi-do-mato fruto

fruto

tuna

ora-pro-nobis

esporão-de-galo, talheira, grutiá

mamãozinho

espinheira-santa,

cancorosa

bibi, biri ou tiririca

batata-doce

melancia-de-porco

abóbora

pepininho-do-mato,

cucumeri

melão-de-são-caetano

batata-cará

maria-preta árvore

cavalinha

folha

folha

folha

folha

folha

raiz

fruto

fruto

folha

folha

fruto

flor

fruto

fruto

fruto

caule folha/caule
folha/flor

folha/flor

raiz tuberosa

flores

raiz tuberosa

ramos

inteiros

ramos

inteiros/raiz

folha/ramos/

salada

ECT7431,

adicionada ao chimarrão/flor cozida

salada

ECT7397,

ECT7610

cozida

salada/refogada

adicionada ao chimarrão/bolos

adicionada ao chimarrão

ECT7468

in natura/saladas/cozida

ECT7470

salada/refogada

ECT7387,

ECT7457

salada/refogada/ raizes

torradas bebida tipo café

ECT7392

salada

salada

salada de folhas, ramos

frescos e raiz

ECT7460

in

natura/suco/schmier/doce/lico

$r$

in natura/schmier/doce/licor

ECT7414

in natura/suco

ECT7417

ECT7437

in natura

in natura/suco

ECT7439

in natura

ECT7481

in natura/salda/refogados

in natura

ECT7427

in natura

adicionada ao chimarrão

ECT7402

bulbo

in natura

ECT7477

sopa/bolinhos/refogados

schmier/geleia/doce

ECT7409, ECT7436

flor empanada/frita

in natura/salada

in natura

túbera aérea

salada/sopa/assada/frita

in natura adicionada ao chimarrão 
ERYTHROXYLACEAE

Erythroxylum argentinum

O.E.Schulz

EUPHORBIACEAE

Manihot esculenta Crantz

FABACEAE

Bauhinia forficata Link

Inga semialata (Vell.)

C. Mart.

Inga sessilis (Vell.) Mart.

Vicia sativa $\mathrm{L}$.

FLACOURTIACEAE

Casearia decandra Jacq.

GESNERIACEAE

Sinningia macrostachya

(Lindl.) Chautems

LAMIACEAE

cocão, cocôn

mandioca, aipim

pata-de-vaca

ingá-feijão, vagem-de-

açúcar

ingá-ferradura, vagem-de-

açúcar, caróbole

ervilhaca, vica

guaçatunga

batata-da-pedra

Hyptis mutabilis (Rich.) Briq. hortelã

Mentha pulegium L.

Plectranthus ornatus Codd

Vitex megapotamica

(Spreng.) Moldenke

MALVACEAE

Hibiscus rosa-sinensis L.

Malvaviscus arboreus Cav.

Sida rhombifolia L.

MELASTOMATACEAE

Clidemia hirta (L.) D.Don

MORACEAE

Ficus cestrifolia Schott

Ficus luschnathiana (Miq.)

Miq.

Morus nigra L.

MUSACEAE

Musa $\times$ paradisiaca L.

\section{MYRTACEAE}

Acca sellowiana (O.Berg)

Burret

Blepharocalyx salicifolius

(Kunth) O.Berg

Campomanesia aurea

O.Berg

Campomanesia

guazumifolia (Cambess.)

O.Berg.

Campomanesia xanthocarpa (Mart.) O.Berg

Eugenia involucrata DC.

Eugenia myrcianthes Nied.

Eugenia pyriformis

Cambess.

Eugenia rostrifolia

D.Legrand

Eugenia uniflora L.

Eugenia uruguayensis

Cambess.

Myrcia palustris DC. poejo

boldo, boldo-gambá

tarumã-preto

hibisco

malvavisco

guanxuma, guaxumba

pixirica

figueira-da-folha-miúda

figueira-da-folha-graúda

amora-de-árvore

coração de bananeira

goiaba-serrana,

goiabinha-do-mato,

goiabinha-do-campo

murta

guavirova-do-campo

sete-capotes

guavirova, guabiroba,

fruta-do-arroio, árvore-do- fruto arroio

cerejeira

pêssego-do-mato

fruto

fruto

uvaia

fruto

batinga

fruto

pitanga

fruto

cambuim

guamirim fruto/casca

do caule

raiz

folhas

arilo

arilo

semente

fruto

tubérculo

cozido na água

ECT7474

adicionada ao

chimarrão/sucos/bolos/molho

de carne

adicionada ao chimarrão

adicionada ao chimarrão

in natura

salada

salada

ECT7413

in natura

ECT7403

in natura

ECT7446

in natura/doce

in natura/doce

ECT7406

in natura/suco/schmier/geleia

ECT7450

parte da

inflorescênci

cozido/molho

fruto/pétala

in natura/suco

ECT7380

ECT7408

ECT7482

ECT7384

in natura

ECT7385,

in natura

ECT7429

in natura

ECT7454

in natura/suco/schmier

ECT7475

in natura/suco/schmier/geleia

in natura

in natura/suco/vinagre

in natura

in natura/suco/geleia/licor

in natura

in natura
ECT7420

ECT7484 
Myrcianthes pungens

(O.Berg) D.Legrand

guabijú

pau-ferro

Schott

Plinia cauliflora (Mart.)

kausel

Psidium cattleianum Afzel. ex Sabine

Psidium salutare var.

sericeum (Cambess.)

Landrum

Syzygium cumini (L.) Skeels OXALIDACEAE

Oxalis latifolia Kunth

PASSIFLORACEAE

Passiflora caerulea L.

PHYLLANTHACEAE

Phyllanthus niruri L.

PLANTAGINACEAE

Plantago major L.

POACEAE

Cymbopogon citratus (D.C.)

Stapf.

PODOCARPACEAE

Podocarpus lambertii

Klotzsch ex Endl.

POLYGONACEAE

Rumex acetosa L.

Rumex obtusifolius L.

PORTULACACEAE

Portulaca oleracea L.

\section{RHAMNACEAE}

Hovenia dulcis Thunb.

Scutia buxifolia Reissek

ROSACEAE

Eriobotrya japonica (Thunb.)

Lindl.

Fragaria sp.

Rosa sp.

Rubus imperialis Cham. \&

Schltdl.

Rubus rosifolius Sm.

Rubus urticifolius Poir.

\section{RUTACEAE}

Citrus japonica Thunb.

Citrus limonia Osbeck

Citrus sinensis (L.) Osbeck

Citrus reticulata Blanco

bergamota-comum

Citrus aurantiifolia

(Christm.)

jabuticaba

vermelho

jambolão

vinho

maracujá-do-mato,

maracujazinho

quebra-pedras

pinheiro-manso

azedinha horta galinha

curunilha

moranguinho

rosa

laranjinha-azeda

limão

laranjeira

lima araçá-amarelo, araçá-

fruto

in natura/suco

ECT7401

ЕСT7483

ECT7442

fruto/flor

in natura

fruto

in natura/sucos/geleia/licor

araçazinho-do-campo

trevinho, azedinha-pão-e-

fruto

in natura/suco/schmier/geleia

ECT7386,

ECT7407,

ECT7435

ECT7433

fruto

bulbo/flor/

seiva

fruto

folha

tansagem, tanchagem

folha/

sementes

capim-cidreira, cidreira

folha

epimácio

in natura

língua-de-vaca

folha

folha

beldroega, erva-gorda-da-

folha/ramo

salada

salada

salada/refogada

uva-do-japão, tripa-de-

fruto

casca do

caule

ameixa-amarela, nespera

amora-branca-do-mato

framboeza, amorinha

amora-preta-do-mato

fruto/

semente

fruto

pétala

fruto

fruto

fruto

flores/casca

do fruto

casca do

fruto

folha/casca

do fruto/flor

folha/casca do fruto/flor casca do

fruto/flor in natura/geleia/bebida

fermentada tipo

vinho/licor/vinagre

adicionada ao chimarrão

in natura/doce/suco/licor

in natura/suco/doce/licor

salada

in natura/doce

in natura

in natura flores

doce da casca

doce da casca/licor de casca e flores/folhas e casca

adicionadas ao chimarrão doce da casca/licor de casca e flores/folhas e casca adicionadas ao chimarrão folhas adicionadas ao chimarrão/doce da casca/licor de casca e flores doce da casca/licor de casca e
ECT7432

ECT7458

ECT7611

ECT7399

ECT7391

ECT7456

ECT7461

ECT7473

ECT7452

ECT7400

ECT7418

CT7466

ECT7388 
SANTALACEAE

Jodina rhombifolia (Hook. \& Arn.) Reissek

SAPINDACEAE

Allophylus edulis (A.St.-Hil., A.Juss. \& Cambess.) Radlk. SOLANACEAE

espinheira-santa-de trêspontas, cancorosa

folha

adicionada ao chimarrão

ECT7467

chal-chal, são-joão, chalichali, avum

in natura/molho e caroço

fruto/caroço

torrado

ECT7448

Physalis pubescens $\mathrm{L}$.

Solanum americanum Mill.

Solanum betaceum Cav.

Solanum sisymbriifolium

Lam.

TROPAEOLACEAE

Tropaeolum majus L.

Tropaeolum pentaphyllum

Lam.

$$
\text { fis }
$$$$
\text { tomate-de-árvore, }
$$$$
\text { tamarijo }
$$$$
\text { juá, arrebenta-cavalo, }
$$$$
\text { mata-cavalo }
$$

fruto

fruto

fruto

fruto

capuchinha

crem

URTICACEAE

Urera baccifera (L.)

Gaudich. ex Wedd.

VERBENACEAE

Aloysia gratissima (Gillies \&

Hook.) Tronc.

Citharexylum montevidense

(Spreng.) Moldenke

XANTHORRHOEACEAE

Aloe vera (L.) Burm.f.

ZINGIBERACEAE

Curcuma longa L.

Hedychium coronarium

J.Koenig

Zingiber mioga (Thunb.)

Roscoe

urtigão
erva-santa, colé
tarumã-vermelho
babosa
açafrão, curcuma
lírio-do-brejo, gengibre-
do-banhado
gengibre

folha/flor/
fruto
tubérculo
frutos/raiz/
seiva
folha
fruto
folha
rizoma
rizoma
rizoma

$\begin{array}{ll}\text { in natura } & \text { ECT7425 } \\ \text { in natura } & \text { ECT7395, } \\ \text { in natura/salada } & \text { ECT7404 } \\ \text { in natura } & \text { ECT7383 } \\ & \text { ECT7393, } \\ & \text { ECT7405 }\end{array}$

salada/conserva do fruto

ECT7382

conserva

ECT7443

frutos in natura/raiz cozida na água/seiva in natura

adicionada ao chimarrão

in natura

adicionada a sucos

salada/condimento

salada/condimento

salada/condimento/adicionada ao chimarrão/doce/bolo
ECT7423

ECT7438

ECT7471

ECT7465

Fonte: Elaborada pelos autores.

Dentre as espécies listadas e apontadas pelas agricultoras e agricultores, 78 (60,47\%) são consideradas nativas ou naturalizadas no Brasil e 51 (39,53\%) são exóticas e em sua maioria introduzidas para cultivo com finalidade comercial ou de uso.

Dentre as 30 espécies mais citadas pelas famílias (Figura 2), a predominância é para frutíferas nativas de ocorrência natural em remanescentes de vegetação, áreas de regeneração, matas ciliares, cultivadas em paisagismo da UPF, ou ainda introduzidas em cultivos de SAF's. As 21 famílias entrevistadas afirmam reconhecer ou utilizarem na alimentação Ananas bracteatus (ananás), Butia odorata (butiá), Eugenia uniflora (pitanga), Psidium cattleianum (araçá) e Syagrus romanzoffiana (coquinho).

Partes não convencionais, no caso as folhas de Beta vulgaris (beterraba) e Daucus carota (cenoura) figuram entre as mais citadas no presente trabalho e assim como em Theis et al. (2018), apresentam distintas formas de preparo e consumo dos agricultores sendo utilizadas como ingredientes para elaboração de bolo frito, em sopas ou refogadas. Além destas, espécies exóticas mais citadas foram: Cymbopogon citratus (capim-cidreira), Morus nigra (amora-de-árvore), Citrulus lanatus var. citroides (melancia-de-porco) Matricaria chamomilla (camomila) e Hovenia dulcis (uva-dojapão). 


\section{Revista Thema}

Figura 2 - Lista das 30 espécies de plantas alimentícias não convencionais (PANC) mais citadas como utilizadas ou reconhecidas pelas 21 famílias de agricultoras e agricultores entrevistados e vinculados à Escola Família Agrícola da Região Sul (EFASUL) em Canguçu, Rio Grande do Sul, Brasil.

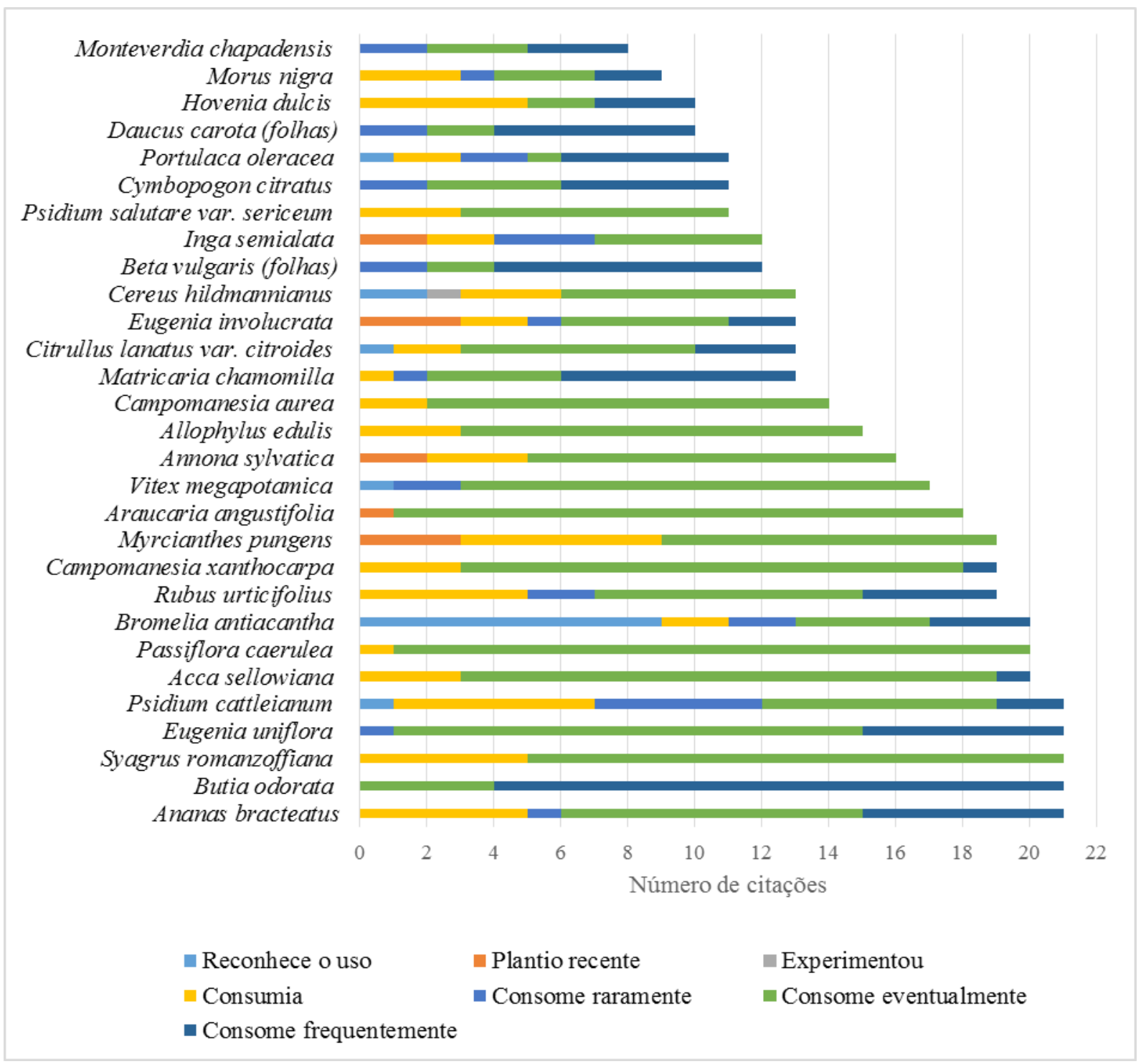

Fonte: Elaborada pelos autores.

Quando analisado as citações das partes de plantas utilizadas para a alimentação, o fruto foi a mais citada, sendo para 56 espécies, seguido de folhas com 38, flor com nove e semente com seis, casca do fruto com cinco, caule com cinco, pétala com quatro, rizoma com quatro, bulbo, raiz e ramos inteiros com três cada, amêndoa, arilo, casca do caule, raiz tuberosa e tubérculo com duas espécies cada e por fim, epimácio (envoltório carnoso e atrativo associado a semente de algumas gimnospermas), palmito, parte da inflorescência, rebentos e tubérculo aéreo com uma espécie cada.

Em relação aos ambientes de ocorrências, a maioria das citações apontaram que as espécies citadas ocorrem predominantemente em mata nativa $(36,74 \%)$, fato que pode ser explicado pelo maior número de frutíferas de ocorrência natural apontadas no presente estudo. Em espaços destinados para hortas ocorrem $18,37 \%$ das PANC, 
seguido de $15,91 \%$ no pátio da UPF, área que compreende o entorno da residência, acessos e entorno de benfeitorias. No ambiente campo de pastoreio, foram apontadas $8,02 \%$ das espécies, lavoura anual 7,5\%, borda de mata nativa, 7,37\%, SAF, 4,40\%, beira de estrada com $0,78 \%$, área de pousio $0,65 \%$ e pomar com $0,26 \%$.

\section{DISCUSSÃO}

A família Myrtaceae foi a que apresentou maior riqueza de espécies de PANC, sendo 18 citadas nas entrevistas, corroborando com estudos florísticos de De Marchi e Jarenkow (2008), para mata ribeirinha do Rio Camaquã que é o limite norte do presente estudo, Soares e Ferrer (2009) para uma floresta ribeirinha do Rio Piratini, Venzke (2012) em áreas pioneiras e Floresta estacional semidecidual da região de Pelotas e Venzke e Martins (2013) em matas ciliares de Arroio do Padre, os quais demonstraram a predominância da família em levantamentos florísticos arbóreos de domínios representativos da área em estudo no presente trabalho.

A família Myrtaceae se destaca entre os entrevistados por apresentar grande potencial alimentício e muitos as cultivam no paisagismo de suas unidades de produção familiares, seja pelo plantio das espécies apreciadas ou por manterem em remanescentes justamente pelo fato de tradicionalmente serem utilizadas na alimentação, fato destacado também por Gomes (2014), que apontou entre outros usos, usos alimentares para o táxon, assim como Theis (2019) e Magalhães (2019), sendo para estes estudos a família de maior riqueza de espécies. Entre as espécies citadas, apenas Syzygium cumini (jambolão) é exótico, com origem na Índia. Outras espécies com distribuição predominantemente ao norte do Estado do Rio Grande do Sul, como Plinia cauliflora (jabuticaba), Eugenia pyriformis (uvaia), Eugenia involucrata (cerejeira) e Campomanesia guazumifolia (sete-capotes) foram citadas, mas também são cultivadas por apreço aos frutos, e principalmente através da doação de mudas por projetos como o de implantação de Quintais Orgânicos de Frutas da Embrapa Clima Temperado, que fomentam a diversidade na agricultura de base familiar e desempenham um reconhecido trabalho para os agricultores da região. (BRASIL, 2016). Neste quesito, há relato de muitas plantas que ainda não frutificaram, pois a inserção de seu cultivo nas UPF é recente. Vale destacar como positiva esta ação, pois além da inclusão destas frutíferas há a valorização e doação das espécies de maior frequência de ocorrência, como Psidium cattleianum (araçá), Eugenia uniflora (pitanga), Campomanesia xanthocarpa (guavirova) e, também, enriquecimento com espécies de menor frequência de ocorrência como Plinia cauliflora (jabuticaba).

A família Asteraceae apresentou 13 espécies, possivelmente pela abundância da família para a região do Bioma Pampa uma vez que Boldrini et al. (2015) relatam o predomínio desta família botânica nas paisagens campestres do Rio Grande do Sul, apresentando a maior riqueza específica. Overbeck et al. (2007), destaca a riqueza de Poaceae e Asteraceae para o bioma. Venzke (2012), aponta como uma das famílias de maior ocorrência, em áreas pioneiras e de regeneração. Asteraceae apresenta potencial de uso predominantemente de folhas devido ao seu hábito herbáceo e arbustivo e a maioria de suas espécies são consideradas naturalizadas conforme o conceito de Schneider (2007) e Moro et al. (2012), que por sua vez, ocorrem espontaneamente e frequentemente são cultivadas, ou mantidas nos espaços de 
cultivos por reconhecimento do potencial de usos diversos como alimento, medicinal ou interesse ecológico, como frequentada por abelhas, insetos predadores, entre outros.

Tanto Myrtaceae quanto Asteraceae são famílias que se destacam para além da riqueza específica no Rio Grande do Sul, pois também apresentam muitas espécies com potencial alimentício. Em levantamento realizado por Kinupp (2007), para a região metropolitana de Porto Alegre, estudo etnobotânico de Theis (2019), na zona rural do município de São Lourenço do Sul e estudo etnobotânico de Magalhães (2019), no contexto da Associação Regional de Produtores Agroecológicos da Região Sul - ARPASUL, que engloba a agricultura familiar de base ecológica dos municípios de Arroio do Padre, Canguçu, Capão do Leão, Morro Redondo, Pelotas e Turuçu, estas foram as famílias mais numerosas apontadas como alimentícias.

Rosaceae apresentou seis espécies, sendo uma de hábito arbóreo e exótica (Eriobotrya japonica). Theis (2019), encontrou oito espécies pertencentes ao táxon no município de São Lourenço do Sul com uso alimentício pelos agricultores e agricultoras.

Rutaceae e Cactaceae apresentaram cinco espécies cada família. A primeira, com espécies cultivadas nas propriedades, tais como laranja, bergamota, lima e limão, conhecidas por serem comercializadas, porém uso de partes não convencionais como cascas permitiram o enquadramento na categoria de PANC. Cactaceae apresenta três espécies nativas do Brasil, outras duas são originárias do México, sendo Hylocereus undatus (pitaya) introduzida recentemente para cultivo como PANC na região e Opuntia ficus-indica (figo-da-índia), introduzido no passado com finalidade ornamental, sendo que o uso alimentar dos frutos nas famílias entrevistadas é recente.

Quanto a parte utilizada, o fruto foi a mais citada, sendo para 56 espécies, estando de acordo com Theis (2019), onde a maioria dos frutos é utilizada através do consumo in natura. Para Durigon et al. (2019), fruto é a parte mais reconhecida pelos agricultores, porém segundo os autores, são as hortaliças as mais utilizadas diariamente na alimentação. Os autores pressupõem que tal fato pode ser explicado pela maioria das espécies de hortaliças encontradas localmente serem espontâneas, abundantes nas UPF e não exigentes de manejo, ou ainda, pelo uso alimentar de partes não convencionais que frequentemente são descartadas. Em seguida as folhas foram citadas como utilizadas na alimentação para 38 espécies, já para Magalhães (2019), elas foram a parte mais citada pelos agricultores entrevistados.

Estudos apontam que na Região Metropolitana de Porto Alegre, 312 espécies possuem potencial de uso alimentício, Kinupp e Barros (2008), já para a metade Sul do RS e mais especificamente no Bioma Pampa não há uma estimativa. Acredita-se que o número de espécies do Bioma esteja além do presente resultado, pois além da temática ser recente no registro destas informações, estudo etnobotânico de Theis (2019), apontou 120 espécies distribuídas em 51 famílias botânicas e Magalhães (2019), encontrou 86 espécies pertencentes a 40 famílias botânicas. Quando somados ao presente estudo, (129 spp. e 55 famílias), representam uma diversidade maior tanto do etnoconhecimento quanto da biodiversidade das PANC, revelando um universo de 67 famílias botânicas e um total de 187 espécies utilizadas pela agricultura familiar regional. Já estudo botânico de cunho prospectivo de PANC 
realizado por Damo et al. (2019), em um sistema agroflorestal de uma única propriedade rural localizada na zona rural do município de São Lourenço do Sul, apontou a ocorrência de 123 espécies distribuídas em 55 famílias botânicas. Um número expressivo corroborando com as pesquisas que revelam o potencial da flora regional.

Segundo Diaz-Betancourt et al. (1999) 10\% da flora de qualquer bioma tem potencial comestível. Levando em consideração estes dados, no Brasil, atingiríamos entre quatro e cinco mil espécies de plantas potencialmente comestíveis. Rapoport et al. (2009) apontam que no Cone Sul, que engloba os países da América do Sul ou parte deles na linha abaixo do Trópico de Capricórnio, se conhecem mais de 500 espécies de "malezas comestibles", muitas das quais são mais apetitosas que plantas convencionais e talvez mais nutritivas. Assim, pesquisas com o foco de resgatar e registrar informações tornam-se necessárias para promover a biodiversidade local e a agricultura de base familiar, visando acrescentar qualidade e diversidade alimentar à mesa.

A revalorização da alimentação vinculada a biodiversidade de plantas espontâneas é uma realidade, de acordo com a FAO, mais de 100 milhões de pessoas na União Europeia - UE, ( $20 \%$ da população) consomem alimentos silvestres. (SCHULP et al., 2014). O programa EU Biodiversa identificou um total de 592 plantas, de 305 gêneros, coletadas na natureza em 17 países da UE e que ao mesmo tempo, estas plantas silvestres fazem parte da história cultural local contribuindo para a identidade e tradições das pessoas. (BACCHETTA et al., 2016). Ainda para Fonseca et al. (2017), vislumbrar uma sociedade com disponibilidade e qualidade de alimentos implica em diversificar a alimentação valorizando a produção local, modificando padrões de consumo, promovendo de forma conjunta a conservação da agrobiodiversidade, a autonomia e a preservação da cultura local.

Para Gomes (2014), os agricultores possuem um grande conhecimento construído individualmente ou acumulado pela experiência dos ancestrais sobre a flora arbórea do local onde vivem. Essa relação é perceptível nos relatos onde agricultoras e agricultores vinculam seus saberes às suas regiões de origens, relacionando-os com o tempo. Esses saberes possibilitam que se valorize a cultura e a identidade das pessoas que lá vivem a fim de melhores condições de vida, valorizando e enriquecendo essa cultura camponesa que historicamente foi e, ainda é menosprezada e subjugada pelas pessoas do meio urbano. (RODRIGUES; BONFIM, 2017; CASTAMAN, 2018).

Por fim, valorizando a biodiversidade, a agricultura familiar local e estimulando a sustentabilidade dos agroecossistemas, Fonseca et al. (2017), apontam que ampliar a diversidade de cultivos, torna-se indispensável para a sustentabilidade dos sistemas de produção de base ecológica, pois quanto maior a diversificação produtiva, menor será a pressão sobre os recursos naturais e consequentemente maior a resiliência do sistema frente às adversidades ambientais, tornando a conservação e o uso da agrobiodiversidade ações complementares. 


\section{CONSIDERAÇÕES FINAIS}

Estudos relacionados a temática PANC são recentes na Região Sul do Rio Grande do Sul e inúmeras iniciativas de levantamentos botânicos e etnobotânicos oportunizadas por instituições como UFPel, EMBRAPA, EFASUL e FURG propiciam o registro, o resgate de seus usos, a valorização da cultura local e a divulgação das informações buscando estimular a agricultura familiar regional, a diversificação da produção e, consequentemente a ampliação das possibilidades de comércio. Nesta linha de raciocínio, Madeira e Botrel (2019), afirmam que as hortaliças PANC podem contribuir para a melhoria na "segurança e soberania alimentar e nutricional" e consequentemente na saúde em geral da população por possuírem características como simplicidade de cultivo, ampla adaptabilidade e rusticidade, e, reconhecidas características nutracêuticas. Também são produtos de origem local e diferenciados, que estimulam a revalorização do tradicional na culinária e na gastronomia, sendo para agricultores de base familiar uma alternativa frente a um mercado cada vez mais competitivo.

Se por um lado o acrônimo PANC popularizou e colocou em discussão uma série de plantas que estavam em desuso, incluindo-as em usos gourmet e apontando inúmeros benefícios, por outro, demonstrou que o conhecimento das populações tradicionais não é arcaico nem desprezível.

A sabedoria destas agricultoras e agricultores revela uma forma de preservação cultural ao longo de gerações dentro de um contexto agrícola predominantemente homogêneo e é semente fértil na disseminação do conhecimento, necessitando ser valorizada, continuada e apoiada por instituições de ensino e pesquisa buscando fortalecer estes sistemas tradicionais e visando assim, produção de alimentos que levem até a mesa do consumidor vida, diversificação alimentar e resiliência para as futuras gerações.

\section{AGRADECIMENTOS}

À Universidade Federal de Pelotas (UFPel), Embrapa Clima Temperado, Escola Família Agrícola da Região Sul (EFASUL), Coordenação de Aperfeiçoamento de Pessoal de Nível Superior (Capes) pelo apoio financeiro à pesquisa e às famílias que se disponibilizaram em participar da pesquisa e abriram as porteiras das propriedades.

\section{REFERÊNCIAS}

ALBUQUERQUE, U. P. Ten important questions/issues for ethnobotanical research Acta Botanica Brasilica, Belo Horizonte, v.33, n.2, apr./jun. 2019.

APG IV. An update of the Angiosperm Phylogeny Group classification for the orders and families of flowering plants: APG IV. Botanical Journal of the Linnean Society, n.181, p.1-20, 2016.

BACCHETTA, L. et al. A manifesto for the valorization of wild edible plants. Journal of Ethnopharmacology, v.191, p.180-187, 2016. 
BOLDRINI, I. I.; OVERBECK, G.; TREVISAN, R. Biodiversidade de plantas. In: PILLAR, V. de P.; LANGE, O. (Ed.). Os campos do Sul. Porto Alegre: Rede campos Sulinos-UFRGS, 2015. p.51-60.

BOTELHO FILHO, F. B. Agricultura familiar e desenvolvimento territorial. Núcleo de Estudos Avançados, Brasília, v.5, n.17, 2005.

BRACK, P. Plantas alimentícias não convencionais. Agriculturas, v.13, n.2, jun. 2016.

BRASIL. Projeto Quintais Orgânicos de Frutas. Pelotas: EMBRAPA Clima Temperado, 2016.

CASTAMAN, A. S.; VIEIRA, J. A.; RADKE, C. L. O contexto atual da educação do campo: o que dizem as pesquisas realizadas. Criar Educação, Criciúma, v.7, n.1, jan./jul. 2018.

CHIVANDI, E. et al. Potential of indigenous fruit-bearing trees to curb malnutrition, improve household food security, income and community health in Sub-Saharan Africa: a review. Food Research International, v.76, part.4, p.980-985, out. 2015.

CORADIN, L.; SIMINSKI, A.; REIS, A. Espécies nativas da flora brasileira de valor econômico atual ou potencial: plantas para o futuro - Região Sul. Brasília: MMA, 2011.

DAMO, A.; HERRMANN, L. K.; DURIGON, J.; BESKOW, G. T. O levantamento florístico de plantas alimentícias não convencionais (PANC) em uma agrofloresta no sul do Brasil. In: CONGRESSO BRASILEIRO DE AGROECOLOGIA, 11., 2019, São Cristóvão. Anais... São Cristóvão: UFS, 2019.

DE MARCHI, T. C.; JARENKOW, J. A. Estrutura do componente arbóreo de mata ribeirinha no rio Camaquã, município de Cristal, Rio Grande do Sul, Brasil. IHERINGIA, Série Botânica, Porto Alegre, v.63, n.2, p.241-248, jul./dez. 2008.

DIAZ-BETANCOURT, M. E. GHERMANDI, L.; LADIO, A.; LOPEZ-MORENO, I. R.; RAEFAELE, E.; RAPOPORT, E. H. Weeds as a source for human consumption. A comparison between tropical and temperate Latin America. Revista de Biología Tropical, San José, v.47, n.3, p.329-338, sep. 1999.

DURIGON, J.; THEIS, J.; SEIFERT, C. A.; VALENTE, C. Hortaliças não convencionais como uma estratégia de sustentabilidade para agricultura familiar no sul do brasil. In: ENCONTRO NACIONAL DE HORTALIÇAS NÃO CONVENCIONAIS, 3., 2019, Curitiba. Anais... Curitiba: Embrapa, 2019.

FAO. Segundo informe sobre el estado de los recursos fitogenéticos para la alimentación y la agricultura en el mundo. Comisión de recursos genéticos para la alimentación y la agricultura. Organización de las Naciones Unidas para la Agricultura y la Alimentación. Roma: Food and Agriculture Organization of the United Nations, 2010.

FAO. Future Smart Food Rediscovering hidden treasures of neglected and underutilized species for Zero Hunger in Asia. Executive summary. Bangkok: Food and Agriculture Organization of the United Nations, 2018. 
FLORA DO BRASIL. Jardim Botânico do Rio de Janeiro. Rio de Janeiro: Instituto de Pesquisas Jardim Botânico do Rio de Janeiro, 2020. Disponível em: http://floradobrasil.jbrj.gov.brl. Acesso em: 7 fev. 2020.

FONSECA, C.; LOVATTO, P.; SCHIEDECK, G.; HELLWIG, L.; GUEDES, A. F. A importância das Plantas Alimentícias Não Convencionais (PANCS) para a sustentabilidade dos sistemas de produção de base ecológica. Cadernos de Agroecologia, v.13, n.1, jul. 2017.

GOMES, G. C. As árvores nativas e o saber local como contribuição à sustentabilidade de agroecossistemas familiares na Serra dos Tapes, RS. 2014. 352 f. Tese (Programa de Pós Graduação Sistema de Produção Agrícola Familiar) - Universidade Federal de Pelotas, Pelotas, 2014.

IBGE. Censo agropecuário 2006. Rio de Janeiro: Instituto Brasileiro de Geografia e Estatística, 2006.

IBGE. Manual técnico da vegetação brasileira: sistema fitogeográfico, inventário das formações florestais e campestres, técnicas e manejo de coleções botânicas, procedimentos para mapeamentos. Rio de janeiro: Instituto Brasileiro de Geografia e Estatística, 2012.

IPNI. The International Plant Names Index. 2020. Disponível em: http://www.ipni.org. Acesso em: 4 fev. 2020.

JOSHI, S. K.; BALLABH, B.; NEGI, P. S.; DWIVEDI, S. K. Diversity, Distribution, Use Pattern and Evaluation of Wild Edible Plants of Uttarakhand, India. Defence Life Science Journal, v.3, n.2, p.126-135, apr. 2018.

KELEN, M. E. B.; NOUHUYS, I. S. V.; KEHL, L. C.; BRACK, P.; DA SILVA, D. B. Plantas alimentícias não convencionais (PANCs): hortaliças espontâneas e nativas. Porto Alegre: UFRGS, 2015.

KINUPP, V. F. Plantas alimentícias não-convencionais da região metropolitana de Porto Alegre, RS. 2007. 590 f. Tese (Programa de Pós-Graduação em Fitotecnia) Universidade Federal do Rio Grande do Sul, Porto Alegre, 2007.

KINUPP, V. F.; BARROS, I. B. I. D. Teores de proteína e minerais de espécies nativas, potenciais hortaliças e frutas. Ciência e Tecnologia de Alimentos, v.28, n.4, p.84657, 2008.

KINUPP, V. F.; LORENZI, H. Plantas alimentícias não convencionais (PANC) no Brasil. Guia de identificação, aspectos nutricionais e receitas ilustradas. Nova Odessa: Instituto Plantarum de Estudos de Flora, 2014.

KISSMANN, G. K. Plantas infestantes e nocivas. 2. ed. São Bernardo do Campo: BASF Brasileira, 1997. Tomo I.

KISSMANN, G. K. Plantas infestantes e nocivas. 2. ed. São Paulo: BASF, 2000. Tomo III.

KISSMANN, G. K.; GROTH, D. Plantas infestantes e nocivas. 2. ed. São Paulo: Basf, 1999. Tomo II. 
LEVY, R. B. et al. Consumo e comportamento alimentar entre adolescentes brasileiros: Pesquisa Nacional de Saúde do Escolar (PeNSE), 2009. Ciência \& Saúde Coletiva, Rio de Janeiro, v.15, n.2, p.3085-3097, 2010.

LORENZI, H. Manual de identificação e controle de plantas daninhas: plantio direto e convencional. 7. ed. Nova Odessa: Instituto Plantarum, 2014.

LORENZI, H.; ABREU MATOS, F. J. Plantas medicinais do Brasil: Nativas e Exóticas cultivadas. Nova Odessa: Instituto Plantarum, 2008.

LORENZI, H.; BACHER, L. B.; LACERDA, M. T. C. Frutas no Brasil Nativas e Exóticas (de consumo in natura). São Paulo: Instituto Plantarum de Estudos da Flora, 2015.

LOVATTO, P. B. As plantas bioativas como estratégia à transição agroecológica na agricultura familiar: análise sobre a utilização empírica e experimental de extratos botânicos no manejo de afídeos em hortaliças. 2012. 392 f. Tese (Programa de Pós-graduação em Sistemas de Produção Agrícola Familiar) - Universidade Federal de Pelotas, Pelotas, 2012.

MADEIRA, N. R.; BOTREL, N. Contextualizando e resgatando a produção e o consumo das hortaliças tradicionais da biodiversidade brasileira. Revista Brasileira de Nutrição Funcional, v.43, n.78, 2019.

MADEIRA, N. R.; SILVA, P. C.; BOTREL, N.; MENDONÇA, J. L. de; SILVEIRA, G. S. R.; PEDROSA, M. W. Manual de produção de hortaliças tradicionais. Brasília: Embrapa, 2013.

MAgAlHãeS, R. S. C. Plantas Alimentícias Não Convencionais (PANC): estudo etnobotânico no contexto da Associação Regional de Produtores Agroecológicos da Região Sul - ARPASUL. 2019. 61 f. Dissertação (Mestrado em Sistemas de Produção Agrícola Familiar) - Universidade Federal de Pelotas, Pelotas, 2019.

MANZINI, E. J. Considerações sobre a elaboração de roteiro para entrevista semiestruturada. In: MARQUEZINE, M. C.; ALMEIDA, M. A.; OMOTE, S. (Org.). Colóquios sobre pesquisa em Educação Especial. Londrina: Eduel, 2003. p.11-25.

MONTENEGRO, S. N. Z.; ZAMBRANO, H. G. N. Lineamientos de consumo y fuentes de obtención de los frutos nativos, pengá (Garcinia macrophylla MART), sachi (Gustavia macarenensis PHILIPSON) y shawi (Plinia sp.) en dos comunidades de la amazonía ecuatoriana. Revista Etnobiología, v.17, n.1, p.61-73, abr. 2019.

MOREIRA, H. J. C.; BRAGANÇA, H. B. N. Manual de identificação de plantas infestantes. Campinas: FMC, 2010.

MORO, M. F. et al. Alienígenas na sala: o que fazer com espécies exóticas em trabalhos de taxonomia, florística e fitossociologia? Acta Botanica Brasilica, São Paulo, v.26, n.4, p.991-999, oct./dec. 2012.

OVERBECK, G. E.; MÜLLER, S. C.; FIDELIS, A.; PFADENHAUER, J.; PILLAR, V. D.; BLANCO, C. C.; BOLDRINI, I. I.; BOTH, R.; FORNECK, E. D. Brazil's neglected biome: the South Brazilian Campos. Perspectives in Plant Ecology, Evolution and Systematics, v.9, p.101-116. 2007. 
PASCHOAL, V.; GOUVEIA, I.; SOUZA, N. S. Plantas Alimentícias Não Convencionais (PANC): o potencial da biodiversidade brasileira. Revista Brasileira de Nutrição Funcional, São Paulo, v.33, n.68, 2016.

PEIXOTO, A. L.; MAIA, L. C. Manual de Procedimentos para Herbários. INCTHerbário virtual para a Flora e os Fungos. Recife: Editora Universitária da UFPE, 2013.

RAPOPORT E. H.; MARZOCCA, A.; DRAUSAL, B. S. Malezas comestibles del cono sur y otras partes del planeta. Buenos Aires: Instituto Nacional de Tecnología Agropecuaria, 2009.

RAPOPORT, E. H.; LADIO, A. Los bosques andino-patagónicos como fuentes de alimento. Bosque, Valdivia, v.20, n.2, p.55-64, 1999.

RODRIGUES, H. C. C.; BONFIM, H. C. C. A educação do campo e seus aspectos legais. In: CONGRESSO NACIONAL DE EDUCAÇÃO, 14., 2017, Curitiba. Anais... Curitiba: PUCPR, 2017. p.1373-1387.

SCHNEIDER, A. A. A flora naturalizada no estado do Rio Grande do Sul, Brasil: herbáceas subespontâneas. Biociências, Porto Alegre, v.15, n.2, p.257-268, 2007.

SCHULP, C. J. E.; THUILLER, W.; VERBURG, P. H. Wild food in Europe: a synthesis of knowledge and data of terrestrial wild food as an ecosystem service. Ecological Economics, v.105, p.292-305, 2014.

SOARES, L. R., FERRER, R. S. Estrutura do componente arbóreo em uma área de floresta ribeirinha na bacia do rio Piratini, Rio Grande do Sul, Brasil. Biotemas, v.22, n.3, p.47-55, set. 2009.

THE PLANT LIST. A working list of all known plant species. 2020. Disponível em: http://www.theplantlist.org/. Acesso em: 8 jan. 2020.

THEIS, J. S. Estudo etnobotânico de plantas alimentícias não convencionais (PANC): saberes e sabores da agricultura familiar em São Lourenço do Sul, RS. 2019. 78 f. Dissertação (Mestrado em Sistemas de Produção Agrícola Familiar) - Universidade Federal de Pelotas, Pelotas, 2019.

THEIS, J. S., HEIDEN, G., DURIGON, J. MAUCH, C. R. Mais desperdiçadas do que desconhecidas: partes alimentícias não convencionais na agricultura familiar. In: ENCONTRO NACIONAL DE PÓS-GRADUAÇÃO, 20., 2018, Pelotas. Anais... Pelotas: Universidade Federal de Pelotas, 2018.

TROPICOS. Tropicos.org. Missouri Botanical Garden. 2020. Disponível em http://www.tropicos.org. Acesso em: 8 jan. 2020.

VENZKE, T. S. Florística de comunidades arbóreas no Município de Pelotas, Rio Grande do Sul. Rodriguesia, v.63, n.3, p.571-578, 2012.

VENZKE, T. S.; MARTINS, S. V. Aspectos florísticos de três estágios sucessionais em mata ciliar em Arroio do Padre, extremo sul do Brasil. FLORESTA, Curitiba, v.43, n.2, p.191-204, abr./jun. 2013. 